- Teledentistry for orthodontic referrals has been successfully used by eight GDP practices in Greater Manchester.

- The benefit to the GDP is availability of specialist orthodontic advice based on clinical photographs +/- radiographs sent via email.

- The benefit to the patient is that they may be saved a hospital visit if their referral is first assessed via teledentistry. This will be particularly helpful if patients live far from the orthodontic provider.

\title{
Teledentistry for screening new patient orthodontic referrals. Part 1: A randomised controlled trial.
}

\author{
N. A. Mandall, ${ }^{1}$ K. D. O'Brien, ${ }_{1}^{2}$ J. Brady, ${ }^{3}$ H. V. Worthington ${ }^{4}$ and L. Harvey ${ }^{5}$
}

Objective The primary aim was to evaluate the validity of a teledentistry system for screening new patient orthodontic referrals. The secondary aims were to evaluate whether the teledentistry system affected i) referral rates ii) inappropriate referral rates iii) number of failed appointments. Study design Randomised controlled trial.

Sample Fifteen dental practices in Greater Manchester, UK, were randomly allocated to either a teledentistry test group $(n=8)$ or a control group $(n=7)$. They referred 327 patients over a 15 month period.

Method Practitioners in the test group referred patients to one of two consultant orthodontists via a 'store and forward' teledentistry system consisting of photographs sent as email attachments. The decision to accept or not accept a referral on this basis was compared with the same decision choice when the same patient was subsequently seen on a new patient clinic. This measured the validity of the system with the clinic's decision used as the gold standard. Patients in the control group were referred using the usual letter system. Referral rates, inappropriate referrals and number of failed appointments were then compared between the teledentistry and control groups.

Results The sensitivity (true positive value) of the teledentistry system was high at 0.80 with a positive predictive value of 0.92 . The specificity (true negative value) was slightly lower at 0.73 with a negative predictive value of 0.50 . The inappropriate referral rate for the teledentistry group was $8.2 \%$ and for the controls $26.2 \%(p=0.037)$. There was no statistically significant difference in clinic attendance between teledentistry and control groups $(p=0.36)$.

Conclusions Teledentistry is a valid system for positively identifying appropriate new patient orthodontic referrals. However, there is a risk that a patient is not accepted on the teledentistry system who would benefit from a full clinical examination. Teledentistry could be a significant factor in reducing the inappropriate referral rate. Patient participation in a teledentistry system does not appear to mean they are any more likely to attend their hospital appointment.

\footnotetext{
$1^{*}-40$ rthodontic Department, University Dental Hospital of Manchester, Higher Cambridge Street, M15 6FH

*Correspondence to: Dr N.A. Mandall

Email nicky@fs1.den.man.ac.uk
}

\section{Refereed paper}

Accepted 21 October 04

DOI: $10.1038 /$ sj.bdj.4812930

$\odot$ British Dental Journal 2005; 199: 659-662

\section{INTRODUCTION}

The main aim of this trial was to evaluate whether a teledentistry system would be a valid method of screening new patient orthodontic referrals and whether this system would reduce the inappropriate referral rate. The validity of such a system is important because clinical decision making through electronic means may fall below established standards of care. ${ }^{1}$ Furthermore, there is a lack of research investigating the validity of telemedicine applications ${ }^{2,3}$ despite a number of publications outlining principles of its set-up and evaluation. ${ }^{4-7}$

This trial proposed to take a step back from full diagnosis and treatment planning via teledentistry and planned only to screen new patients for the appropriateness of their referral. A screening system was used because, on average, the wait for a new patient consultation was 4.6 months (range 0-24 months). ${ }^{8}$ Additionally, 20-45\% of patient referrals are inappropriate and increase waiting times unnecessarily. ${ }^{9,10}$

Common reasons for inappropriate referrals were reported as mild malocclusion, referral too early, and poor oral hygiene. ${ }^{9}$ GDPs may often also feel pressurised by parents to refer this type of patient for an opinion, although they themselves realise that the patient is unlikely to receive treatment. The factors outlined above should be easy to assess from clinical photographs and an orthodontic opinion may be obtained quickly thus reassuring patients without the need for a hospital visit. As a result, a 'store and forward' email-based teledentistry system to screen new patient referrals became the focus of this study.

\section{SAMPLE}

\section{Background information}

An initial study was carried out to determine which dental practices referred more than seven patients per year to one of two hospital departments (Hope Hospital, Salford, and University Dental Hospital of Manchester). Thirty-five practices were identified who referred on average 19 patients (range 8-17). The inappropriate referral rate for all referrals to Hope Hospital over one year was found to be $38 \%$.

\section{Sample size calculation}

The sample size was based on the proportion of inappropriate referrals decreasing from $38 \%$ to $10 \%$ during the trial (0.38-0.10). A sample of 15 referrals (on average) per year from each of 16 
practices (eight in the test and eight in the control groups) would have $>90 \%$ power to detect this reduction, assuming that the intraclass correlation coefficient was 0.05 . This resulted in a total sample size for the study of 240 referrals over a one year period.

\section{METHOD}

\section{Practice recruitment}

The 35 practices referring more than seven patients per year were approached to take part in the trial. Sixteen practices responded, however, one practice in the control group withdrew before the trial started.

\section{Random allocation method}

Dental practices and thus GDPs within practices were randomly allocated, using a random-number table, to teledentistry test group or control. Agreement to participate in the trial was obtained before random allocation to ensure that GDPs did not agree to participate based on the computer equipment donated for the trial.

\section{Interventions and their timing}

Written parent and child consent was obtained by the GDPs for all children participating in the trial.

\section{i) Teledentistry test group}

The following patient information was sent to the consultant orthodontist via email with the photographs downloaded from a digital camera as JPEG files.

- Patient name, date of birth and gender

- Patient complaint, medical and dental history

- Overjet (mm)

- Presence of mandibular displacement on closure

- Extra oral frontal and lateral facial photographs

- Intra oral anterior, right and left buccal photographs in occlusion

- Any available radiographs were mounted on a light box and photographed.

Thus the teledentistry method used in this study was 'store and forward'. The orthodontist received an email with attached photographs that could be read outside and away from the clinic. GDPs were trained onsite by our IT consultant and were also given written instructions. IT support was given during the study by telephone and, in two cases, by a practice visit.

\section{ii) Control group}

Once this information had been forwarded, GDPs also referred the patient by conventional referral letter to ensure that the patient was also seen on a new patient clinic. The same orthodontist who assessed the email referral saw the patient on clinic a minimum of eight weeks later. Patients in control practices were referred to the orthodontist by letter in the conventional way.

\section{Patient confidentiality}

Access to patient records on the teledentistry system was limited to the two consultant orthodontists participating in the trial. In addition to the usual password protection, the data were encrypted with a double password protected encryption key.

\section{Outcomes}

The primary outcome was whether the orthodontist would accept or not accept an orthodontic referral based on the teledentistry referral and the clinical examination. These two decisions were compared to assess the validity of the teledentistry system. The following secondary outcomes were compared between the test and control groups:
- Number of referrals

- Inappropriate referral rate

- Failure to attend.

If a patient cancelled or failed to attend an appointment but then subsequently attended, the primary outcomes and the inappropriate referral outcome were recorded and included in the data analysis.

\section{Data collection}

Teledentistry data

The orthodontist recorded whether or not they would accept the patient as a new patient referral using the teledentistry system. The reason for not accepting a referral was recorded in both instances. The definition of inappropriate referral used in this study was:

- Poor oral hygiene

- Mild malocclusion

- Referral too early

- Other - including patient did not want treatment, suitable for a specialist, adult requiring routine orthodontics.

\section{Control group}

Clinical data were recorded for all patients in either test or control group. This consisted of attendance and the outcome of the appointment and therefore whether the patient referral would be accepted. In addition, for inappropriate referrals the reason was recorded as previously outlined.

\section{Examiner blinding}

The orthodontists were blinded to whether patients were in the test or control group to minimise any bias in recording clinical outcomes. Blinding was done as follows:

- Test practices were given an unique identification number so subsequent referral letters could not be linked to test or control group.

- GDPs were asked not to indicate any involvement with the trial on the referral letters.

\section{Memory bias}

Taylor ${ }^{5}$ suggested that the same observers should view the cases in the test group and the effects of learning/remembering cases counteracted by having the clinic a couple of months after the teledentistry system is used. This occurred as a result of natural new patient waiting lists and no patient was seen on a clinic within two months of their electronic referral. In addition, trial patients on clinics were integrated at random with non-trial patients.

\section{Statistics}

Simple summary statistics were produced for age, gender and number of referrals in test and control groups. Sensitivity and specificity and kappa values were calculated to assess the validity of the teledentistry system. Failed attendances were compared between test and control using chi square statistic. Inappropriate referral rates were compared using generalised estimating equations (GEE) with a logit link and an exchangeable correlation matrix. These are an extension of logistic regression analysis, which allowed for clustering of patient referrals within practices.

\section{RESULTS}

After 15 months, 80 patients had been referred in the teledentistry group and 247 in the control group. This sample had sufficient power to detect statistically significant differences between test and control groups for inappropriate referral rates.

Figure 1 summarises the number of practices and patients participating in the trial. The mean age and gender of the patients is summarised in Table 1 and there were no statistically significant differences between test and control groups for these variables. 


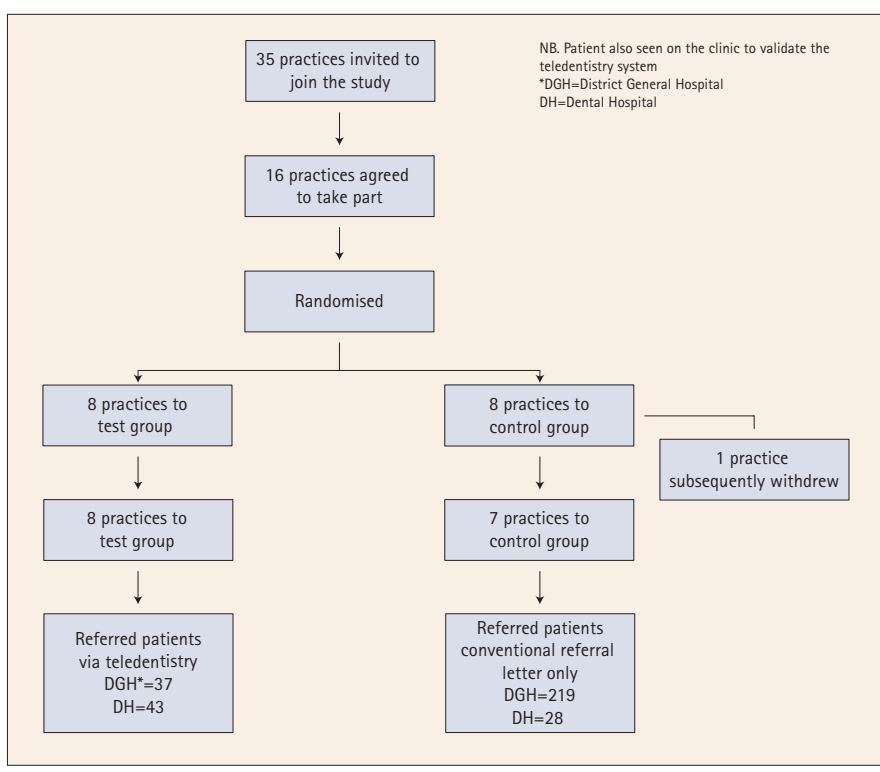

Fig. 1 Number of practices and patients participating in the trial

\begin{tabular}{lcc}
\multicolumn{3}{l}{ Table 1 Age and gender of patients in the teledentistry trial } \\
\hline & \multicolumn{1}{l}{ Teledentistry test group } & Control group \\
\hline $\begin{array}{l}\text { Mean age at clinical } \\
\text { consultation } \\
\text { years/months (SD) }\end{array}$ & $13.1(1.8)$ & $13.8(4.3)$ \\
\hline $\begin{array}{l}\text { Gender (\%) } \\
\text { Male }\end{array}$ & $34(42.5)$ & $101(40.9)$ \\
Female & $46(57.5)$ & $146(59.1)$ \\
\hline
\end{tabular}

Table 2 The inappropriate referral rate in the teledentistry and control groups where patients who would have been accepted as a result of the teledentistry system were selected

\begin{tabular}{lll}
\hline Appropriate referral (\%) & Test & Control \\
\hline Inappropriate referral $(\%)$ & $45(91.8)$ & $152(73.8)$ \\
\hline
\end{tabular}

Table 3 Comparison of first appointment attendance between teledentistry
and control groups
\begin{tabular}{lll}
\hline Attended $(\%)$ & $66(82.5)$ & $192(77.7)$ \\
\hline Did not attend ${ }^{*}(\%)$ & $14(17.5)$ & $55(22.3)$ \\
\hline *Failed and cancelled first appointments combined \\
Chi square P value $>0.05(1 \mathrm{df})$
\end{tabular}

\section{Validity of the teledentistry system}

A cross tabulation of the decision to accept a referral for the teledentistry group is shown in Table 2 . The sensitivity of the system to screen referrals (true positive or (yes/yes agreement) was 0.80 . The specificity of the system (true negative or no/no agreement) was 0.73. Appendix 1. This showed that teledentistry was valid for screening orthodontic referrals. In other words, the decision whether to accept a referral based on teledentistry information was similar to seeing the patient face to face. However, great care still needs to be taken if a patient is refused an appointment because the screening system suggests they should not be seen. Such a patient may not receive full orthodontic assessment when they should have received it. This potentially occurred in 11 cases during this trial.

Thus the positive predictive value of the teledentistry system to screen new referrals was high at 0.92 . However the negative predictive value was lower at 0.50 . This reflected the finding that half of the 22 children not accepted on the teledentistry system, would have been accepted when seen on clinic (Appendix 1).

This important caveat is reflected in the Kappa score, was calculated as 0.46 (standard error 0.12) and reflects the clinician agreement between the decision to accept a new patient based on teledentistry versus conventional clinical information. A score of 0.46 reflects moderate agreement. ${ }^{10,11}$

\section{Inappropriate referral rate}

Patients who would have been accepted as new patient referrals via the teledentistry system were selected and compared with the control group (Table 2). The inappropriate referral rate was lower in the test group (8.2\%) compared with the control group (26.2\%) $(\mathrm{P}=$ $0.037)$.

\section{Hospital appointment attendance}

The data for failed and cancelled appointment were combined into a non-attendance variable. Comparison of attendance between test and controls is shown in Table 3. There was no difference between attendance for the first appointment between teledentistry and control groups $(\mathrm{P}=0.36)$.

\section{DISCUSSION}

The results of this study suggest that teledentistry is a good way of positively identifying patients who should be referred to a consultant orthodontist. Therefore, a valid, efficient and time saving clinical screening method has been identified. These results support the findings of Davies and Stephens. ${ }^{11,12}$ They found no discernable differences in intra-examiner consistency for treatment need, extraction decision or type of appliance when using handheld records or video-conferencing technology.

However, our results did highlight a disadvantage with a teledentistry system in that some cases not accepted for care from electronic data were subsequently accepted as new patient referrals following a full clinical examination (11 out of 56 (19.6\%) of patients). An explanation for this may have been that a patient could improve their oral hygiene between the teledentistry referral and being seen on a clinic, however, the results of this study do not support this hypothesis.

Importantly, a significant minority of patients may not be accepted as new patients after electronic screening, but may benefit from a full clinical examination. A way of addressing this problem could be to accept the new patient referrals in cases where there is uncertainty in accepting the patient based on teledentistry information. Thus, it would be beneficial not to be too selective when screening new patient referrals with this method.

The kappa score for intra-clinician agreement between teledentistry and clinical information was moderate but still higher than other reports of clinical decision-making. ${ }^{12,13-15}$ Therefore, it could be suggested that agreement between screening decisions made from teledentistry and clinical information is acceptable in the light of generally poor examiner agreement for orthodontic decision-making.

\section{The number of patients referred and their attendance}

At the start of the trial, one practice withdrew from the study and there could have been a number of possible reasons for this. The GDP may have felt he did not have enough time to participate - consent procedures can take 10 minutes per patient. Alternatively, staff training, having enough potential suitable referrals, or workload may have caused them to withdraw. Additionally, it was not as easy to give an incentive to GDPs in the control group, as they did not receive computer and camera equipment given to the test practices.

There were statistically significantly more patients referred in the control group for the district general hospital compared with the teaching hospital (Table 2). This cannot be explained by the number 
of practices in each group as there were only seven practices in the control group but eight in the teledentistry group. Other possible reasons might include:

- The teledentistry system reducing the number of referrals because of extra clinical time required to take photographs and send the email referral.

- A number of patients who did not participate in the trial were referred by practices in the teledentistry group. Unfortunately, as this only accounted for eight patients, it does not explain the large differences between groups.

- One practice in the control group had five dentists and routinely referred more patients than the other practices.

When attendance is considered, we initially thought that patients agreeing to take part in the teledentistry aspect of the trial might be more motivated towards orthodontic treatment and, therefore, more likely to attend for their appointment. However, this hypothesis was not borne out in this trial.

\section{Inappropriate referrals}

The finding that the teledentistry system could potentially reduce the inappropriate referral rate to $8 \%$ is clinically important. Previous reports have suggested an inappropriate referral rate of around 45\% but interestingly the rate in the control group in this study was only $26 \%$. There is no reason to expect the control group data to be any different compared with previous literature. However, it is possible that because the control dentists knew they were taking part in a study, their referral pattern could have been different.

A statistically significantly lower inappropriate referral rate associated with teledentistry has widespread service implications. Previously up to $45 \%$ of clinic appointments may have been taken up with inappropriate referrals resulting in appropriately referred new patients waiting longer to be seen. There is thus a huge potential advantage to shorten new patient waiting times by screening out inappropriate referrals using teledentistry.

\section{CONCLUSIONS}

Teledentistry is a valid system for positively identifying appropriate new patient orthodontic referrals. However, importantly there is a risk that a patient is not accepted on the teledentistry system but would benefit from a full clinical examination. This highlights a potential flaw in the validity or accuracy of teledentistry to screen orthodontic referrals.

Teledentistry could potentially be a significant factor in reducing the inappropriate referral rate. This would significantly reduce waiting times for orthodontic referrals. Patient participation in a teledentistry system does not appear to mean they are more likely to attend their hospital appointment. Additionally, a reduction in number of referrals attributable to the use of the system cannot be reliably concluded. GDP perception of teledentistry was also evaluated and will be reported in the part II study.

The authors acknowledge the support of the British Dental Association Shirley Glasstone-Hughes Award, The British Orthodontic Society Houston Research Scholarship, GlaxoSmithKline, The Booth Charities, BNFL, The Roger Clutterbuck Charitable Trust, The Dalmia Charitable Foundation. Thanks to all the GDPs and practice staff who participated in the trial and Dr John Wright for setting up the teledentistry system

1. Jerrold L. Litigation, legislation and ethics. The problem, electronic data transmission and the law. Am J Orthod and Dentofacial Orthop 1998; 113: 175-178.

2. Wallace $\mathrm{S}$, Wyatt J, Taylor P. Telemedicine in the NHS for the millennium and beyond. Postgrad Med J 1998; 74: 721-728.

3. Currell $R$, Urquhart $C$, Wainwright $P$, et al. Telemedicine versus face to face patient care: effects on professional practice and healthcare outcomes. The Cochrane Library 2002 Issue 1.

4. Yellowlees P. Successful development of telemedicine systems-seven core principles. $J$ Telemed Telecare 1997: 3: 215-222.
5. Taylor P. A survey of research in telemedicine. I: Telemedicine systems. J Telemed Telecare 1998; 4: 1-17.

6. Anonymous. Telemedicine: an overview. Health Devices 1999; 28: 88-103.

7. Cook J, Mullings $C$, Vowles $R$, et al. Online orthodontic advice: A protocol for a pilot teledentistry system. J Telemed Telecare 2001; 7: 324-333.

8. Russell J, Pearson A, Bowden D J A, et al. The consultant orthodontist service 1998 survey. BrDent J 1999; 187: 149-153.

9. O'Brien K D, McComb J L, Fox N, et al. Do dentists refer orthodontic patients inappropriately? Br Dent J 1996; 181: 132-136.

10. Bowden D, Pender N, Hussain J, et al. An attempt to influence the referral of orthodontic patients to hospital orthodontic departments. Health Trends 1996; 28: $67-70$.

11. Landis J R, Koch G C. The measurement of observer agreement for categorical data. Biometrics 1977; 33: 159-174

12. Davies J. The use of video conferencing technology in orthodontic treatment planning. 1999; MSc thesis. University of Bristol.

13. Ribarevski $R$, Vig P, Vig K D, et al. Consistency of orthodontic extraction decisions. Eur J Orthod 1996; 18: 77-80.

14. Lee R, Macfarlane T, O'Brien K D. Consistency of orthodontic treatment planning decisions. Clin Orthod Res 1999: 2: 79-84.

15. Mandall N A, Bearn D, Chadwick $S$, et al. Are photographic records reliable for orthodontic screening? J Orthod 2002; 29: 125-127.

Statistical Appendix 1: The decision to accept the referral based on teledentistry information and clinical information

\begin{tabular}{lll}
$\begin{array}{l}\text { Teledentistry decision } \\
\text { to accept the referral }\end{array}$ & $\begin{array}{l}\text { Clinic decision to accept the referral } \\
\text { Yes }\end{array}$ & No \\
\hline Yes & 45 & 4 \\
\hline No & 11 & 11 \\
\hline
\end{tabular}

Sensitivity or "yes/yes agreement to accept the referral of teledentistry vs clinical information $=0.80$.

Definition "sensitivity": the proportion of truly diseased persons in the screened population who are identified as diseased by the screening test (teledentistry system). Sensitivity is the measure of the probability of correctly diagnosing a case or the probability that any given case will be identified by the test.

Specificity or "no/no agreement to accept the referral teledentistry vs clinical information 0.73 .

Definition "specificity": The proportion of truly non-diseased persons who are so identified by the screening test. It is the measure of the probability of correctly identifying a non-diseased person with a screening test.

Positive predictive value of the teledentistry system to screen new referrals=0.92.

Negative predictive value of the teledentistry system to screen new referrals $=0.50$.

\section{Statistical Appendix 2}

Generalised estimating equations resulted in an odds ratio of 0.25 (95\% confidence intervals 0.068-0.92), which suggests that the test group had $75 \%$ fewer inappropriate referrals than the control group. This is derived by considering that the odds of an inappropriate referral is the ratio of the probability of it occurring to it not occurring. The odds ratio is the ratio of the odds of an inappropriate referral in the teledentistry group to the odds of an inappropriate referral in the control group. An odds ratio of 1.00 would have meant that the test and control group were equally likely to produce inappropriate referrals.

Statistical Appendix 3: Reasons given by the orthodontists for inappropriate referral cases using teledentistry and clinical information

$\begin{array}{llll}\text { Clinical reason for } & \text { Teledentistry reason for inappropriate referral } \\ \text { inappropriate referral } & \text { Poor } \mathrm{OH} & \begin{array}{l}\text { Mild } \\ \text { malocclusion }\end{array} & \text { Too early }\end{array}$

Poor $\mathrm{OH}$

Mild malocclusion 3

Too early

Other 1 2

*0ther $=$ suitable for specialist.

The reasons for the inappropriate referrals where teledentistry and clinical decision to accept the patient were both "no" are shown. This data suggests that within clinician agreement for why a referral would not be accepted is very consistent. 